\title{
Descriptive Analysis of the Medical Consultations Conducted in the Dental School of Kerman University of Medical Sciences
}

\author{
Atai $Z^{1}$, Navabi $\mathbf{N}^{2 *}$, Khoshroo $\mathrm{SMR}^{3}$, Asadi $\mathbf{L}^{4}$ \\ ${ }^{1}$ Associate Professor, Oral Medicine Dept, Kerman Dental School, Kerman, Iran \\ ${ }^{2}$ Associate Professor, Social Determinants on Oral Health Research Center,Kerman University of Medical Sciences,Kerman, Iran \\ ${ }^{3}$ Assistant Professor,Biology Dept, Kerman Branch, Islamic Azad University, Kerman, Iran \\ ${ }^{4}$ Dentist
}

\begin{tabular}{l} 
ARTICLE INFO \\
\hline Article Type \\
Original Article \\
\hline Article History \\
Received: June 2016 \\
Accepted: Sep 2016 \\
ePublished: Jan 2017
\end{tabular}

Keywords:

Medical consultation,

Dental clinics,

Patient care planning

\begin{abstract}
Background and Aim: Medical consultations have an important role in dental diagnosis and treatment planning, and are indicated for patients with uncertain medical histories. The purpose of the present study was to evaluate the medical consultations conducted for a group of patients in need of dental treatments.

Materials and Methods: In this cross-sectional study, 173 medical consultation requests were reviewed at the diagnosis and oral medicine department of the dental school of Kerman University of Medical Sciences during 2014-2015. The demographic data, reasons for consultation, and medical precautions were descriptively analyzed.

Results: The mean age of the patients was $37.3 \pm 6.5$ years, and $64.2 \%$ of the subjects were females. The main reasons for medical consultations were hypertension $(28.9 \%)$ and cardiovascular assessment (19.7\%). The main dental concerns included the need for preoperative antibiotic prophylaxis $(7.5 \%)$ and the use of local anesthesia without epinephrine (19.7\%).

Conclusion: Medical consultations reduce the medical risks associated with dental procedures and unnecessary antibiotic prophylaxis. Good communication between dentists and physicians is essential for satisfactory patient care
\end{abstract}




\section{Introduction:}

Dental profession has dramatically changed over the past two decades, and it is no longer merely limited to practical techniques and approaches. As a result of medical advances and various available treatments, fewer patients lose their lives. Due to the increased number of dental patients who also suffer from systemic diseases, dentists must have an acceptable level of knowledge about systemic diseases. ${ }^{(1-3)}$ The statistics show that during the course of his/her professional career, a dentist visits at least 100 asthmatic patients. Furthermore, a dentist visiting 2000 adult patients, is expected to meet at least 130 patients with chronic obstructive pulmonary disease, 100 patients with peptic ulcer disease, 220 patients with chronic renal failure, 100 patients taking corticosteroids with the possibility of adrenal suppression, and 3-4 patients with epilepsy. ${ }^{(4-6)}$

Patients who have their first dental visit must consult with specialists in order to clarify different aspects of their general health status including the ones which are of ambiguity for the dentist upon checking the background or performing physical examination. ${ }^{(7-8)}$ Medical consultation is scientifically and legally considered as an important step in planning dental treatment for patients with systemic problems. ${ }^{(1)}$ Many systemic diseases or their treatments necessitate changes in dental treatment plans. Furthermore, lack of due consideration and specialized care in dental treatments may cause serious complications and result in various problems during dental treatment. ${ }^{(9-12)}$

Due to the increasing number of dental patients who also suffer from chronic medical problems as well as the potential risks that dental treatments can cause, medical consultation seems necessary prior to dental treatment. Successful dental treatments require a strong cooperation between dentists and physicians, and consultation is of utmost importance in the establishment of this cooperation. Proper medical consultations are important in terms of offering suitable treatment services, as well as in terms of legal issues, and determining the pattern of current medical consultations would contribute to improving the aforementioned cooperation in the future. Therefore, the present study aimed to determine the specifications of the medical consultations performed in the department of oral medicine of the dental school of Kerman University of Medical Sciences.

\section{Materials and methods:}

In this descriptive study, sequential sampling method was used. The study population consisted of the patients attending the dental school of Kerman University of Medical Sciences from September 23, 2014 to September 23, 2015. In this dental school, all patients are initially examined and their specifications are filed and recorded. All the examinations are conducted under the supervision of oral medicine specialists, and when medical consultations are needed, they are conducted with the cooperation of the specialists using pre-prepared forms. The consultation meeting is transcribed by a dental student or an oral medicine resident and its contents are finally confirmed by the oral medicine specialist. The printed pre-prepared medical consultation forms were used as research instruments for data collection. Each form consisted of three sections: ${ }^{(1)}$ Patient's demographic information (name, date of examination, age, sex, case number, marital status, and occupation); (2) Consultation reason, which is explained, stamped and signed by the dentist; (3) Specialists' comment, which is explained, stamped, and signed by the specialist. (13) After conducting the consultations, the forms completed by the physicians and the forms responded by the patients were transferred to the department of oral medicine, and copies of them were kept by a senior dental student in a separate file until the end of the study. All the medical consultations carried out during the study period were included in this research. Those patients who did not return or did not participate in medical consultations were excluded. The participants' personal information was confidential. The present study was approved by the department of science and technology of Kerman University of Medical Sciences with the ethics code number of ir.kmu.rec.1394.727.

The data were analyzed using SPSS software 
version 21 (IBMC Corp., Armonk, NY, USA). Descriptive statistical analysis was performed through calculating the frequency and the percentage of frequency.

\section{Result:}

During the study period, 6237 patients attended the dental school of Kerman University of Medical Sciences. However, only 173 cases $(2.77 \%)$ required medical consultations. Table 1 shows the distribution of the age and gender of the consulted participants. The age range of the participants was between 10 to 90 years and was divided by 10 -year-old age groups. The highest and lowest frequencies of the conducted consultations were associated with the age groups 20-29 years and 80-90 years, respectively. The mean age of the participants was $37.3 \pm 6.5$ years. According to Table 1, $64.2 \%$ of the patients were females.

Table 1- Frequency distribution of the consulted patients based on age and gender

\begin{tabular}{cc}
\hline Age (Years) & Frequency (Percentage) \\
\hline $10-19$ & $20(11.5)$ \\
\hline $20-29$ & $35(20.3)$ \\
\hline $30-39$ & $26(15)$ \\
\hline $40-49$ & $25(14.5)$ \\
\hline $50-59$ & $32(18.5)$ \\
\hline $60-69$ & $25(14.5)$ \\
\hline $70-79$ & $7(4)$ \\
\hline $80 \leq$ & $3(1.7)$ \\
\hline Total & $173(100)$ \\
\hline Gender & $62(35.8)$ \\
\hline Male & $11(64.2)$ \\
\hline Female & $173(100)$ \\
\hline Total
\end{tabular}

Table 2 represents the distribution of the conducted consultations based on systemic diseases. The first and second highest frequencies of consultations were related to hypertension (28.9\%) and heart diseases (19.7\%), respectively. Only one case of infectious disease was consulted.

Table 3 presents the frequency distribution of the remarks delivered by the specialists. The remarks included patient's need for special considerations, antibiotic prophylaxis or use of local anesthesia without epinephrine.
Table 2 - Frequency distribution of medical consultations based on systemic diseases

\begin{tabular}{cc}
\hline Systemic Disease & Frequency (Percentage) \\
\hline Cardiovascular & $34(19.7)$ \\
\hline Hypertension & $50(28.9)$ \\
\hline Endocrine & $12(6.9)$ \\
\hline Hematologic & $15(8.7)$ \\
\hline Neurologic \& Psychologic & $22(12.7)$ \\
\hline Liver \& Gastrointestinal & $12(6.9)$ \\
\hline Pulmonary & $4(2.3)$ \\
\hline Ear , Nose \& Throat & $2(1.2)$ \\
\hline Skin & $2(1.2)$ \\
\hline Urogenital & $4(2.3)$ \\
\hline Autoimmune & $9(5.2)$ \\
\hline Pregnancy & $6(3.5)$ \\
\hline Infectious & $1(0.6)$ \\
\hline Total & $173(100)$ \\
\hline
\end{tabular}

Table 3- Frequency distribution of the medical remarks based on the physician's advice

\begin{tabular}{cc}
\hline Special & Frequency (Percentage) \\
Consideration(s) & \\
\hline No & $73(42.2)$ \\
\hline Yes & $100(57.8)$ \\
\hline Total & $173(100)$
\end{tabular}

\begin{tabular}{cc}
\hline \multicolumn{2}{c}{ Antibiotic Prophylaxis } \\
\hline No \\
\hline Yes \\
\hline Total & $160(92.5)$ \\
\hline Local Anesthesia without Epinephrine & $173(100)$ \\
\hline No & \\
\hline Yes & $139(80.3)$ \\
\hline Total & $34(19.7)$ \\
\hline
\end{tabular}

\section{Discussion:}

This descriptive study evaluated the medical consultations $(2.77 \%)$ carried out for the patients attending the dental school of Kerman University of Medical Sciences. Due to involvement of various factors (including differences in the prevalence of systemic diseases, differences in the quality control of these diseases in different areas, as well as the differences across skills and knowledge of dentists who conduct the initial visits with the patients, and their disagreement on the necessity of medical consultations), no particular figure or range can be considered for medical consultations. Laurence evaluated the 
dentists' beliefs regarding the importance of regular screening for systemic diseases, and stated that the highest frequencies in terms of importance of screening were reported for hypertension (85.8\%), cardiovascular diseases $(76.8 \%)$ and diabetes mellitus $(76.6 \%){ }^{(14)}$

It seems that, regardless of the values related to the conducted consultations, dentists should be familiar with the important role of medical consultation in the detection of undiagnosed systemic diseases, and that they should not consult with physicians solely to obtain a license and to confirm the treatment for patients with systemic diseases. It seems that one of the reasons that general practitioners fail to conduct medical consultations is the referral of patients with systemic diseases to dental specialists. In a study by Cottrell et al, the majority of dental patients with systemic problems were referred to maxillofacial surgeons. ${ }^{(15)}$

In the present study, the highest frequency of medical consultations was recorded for the age group 20-29 years; however, it is commonly expected that the elderly suffer more from chronic medical problems. Jainkittivong, et al. claimed that about $80 \%$ of the patients above 60 years of age who had requested dental treatments were suffering from systemic problems, and the continuous use of various drugs also increases with age. ${ }^{(16)}$ It is likely that the higher frequency of consultations conducted for the age group 29-20 years in the current study is because of the greater concern of dentists about the symptoms expressed by the patients at the time of medical history recording.

In the present study, the majority of the patients were females $(64.2 \%)$, consistent with the results of studies by Jainkittivong et al. and Hatipoglu et al. ${ }^{(16,17)}$ Higher life expectancy and higher number of dental visits among women are the probable reasons for this finding.

In this study, most medical consultations were conducted for hypertension and heart diseases. This finding is consistent with those found by Jainkittivong et al. ${ }^{(16)}$ Similarly, most dental consultations in the study by Hatipoglu et al were those between cardiologists and dentists. ${ }^{(16,17)}$ This can be due to the high prevalence of hypertension and cardiovascular dis- eases, as well as the dentists' concerns about serious heart complications such as dangerous arrhythmias and syncope during dental treatment.

It is worth mentioning that in the present study, consultations for psychological problems were of relatively high frequency $(12.7 \%)$, while only one consultation for infectious diseases was conducted. These may also originate from the dentists' concerns about the risk of seizures during dental treatment of patients with neurological disorders, dentists' adequate information about diseases such as hepatitis, as well as specified protocols used in the diagnosis and treatment of infectious diseases. ${ }^{(1,18)}$

In the current research, $57.8 \%$ of the doctors recommended special considerations for patients. This value was equal to $1.32 \%$ and $55.3 \%$ in studies by Jainkittivong et al and Hatipoglu et al, respectively. ${ }^{(16,17)}$ The value obtained in the second study is close to the one in this study. In the current research, antibiotic prophylaxis was recommended in $7.5 \%$ of consultations, while $19.7 \%$ of the doctors also recommended the use of local anesthesia without epinephrine. These values were $32 \%$ and $20.4 \%$, respectively for antibiotic prophylaxis and use of local anesthesia without epinephrine in the study by Jainkittivong et al. ${ }^{(16)}$ Although Jainkittivong et al evaluated these two aspects from the perspective of the dentists' concerns, the results of the present study are associated with the physicians' emphasis on these two aspects. ${ }^{(16)}$

Jainkittvong et al and Hatipoglu et al stated that a new medical diagnosis and the initiation of treatment were considered for $8 \%$ and $16.35 \%$ of the patients after consultations. ${ }^{(16,17)}$ In the present study, such values were not derived from the data.

In the present study, all the answers to the consultations were handwritten, and this can be considered as a limitation, since illegible handwritings cause probable confusions. Another non ideal observation was the overuse of abbreviated medical terminology by respondent physicians, as dentists may not be familiar with these abbreviations. ${ }^{(18)}$

Geist et al stated that the use of a standard and structured medical consultation form can improve the quality of the responses to dentists' medical consultations. ${ }^{(19)}$ Ahsen et al. recommended the use of training video clips in order to improve the quality of medical consultation, which is considered as a type of skill. (20) The results of this study suggest that more efforts should be made to standardize medical consultation forms, and a more accurate program should be reinforced to teach the indications for medical consultation 
to dentists. Medical consultation forms should be standardized. The basics of medical consultation are usually communicated during general dentistry courses; however, lack of sufficient practice may decrease the quality of medical consultations conducted by dentists.

\section{Conclusion:}

Dental management of medically-compromised patients may encompass different risks. The data from the present study reinforce the necessity of medical consultation for individuals with systemic diseases. The consultation process may affect the diagnosis and treatment plan.

\section{Acknowledgments:}

This study was financially supported by Kerman University of Medical Sciences (Research No: 94/213). The authors wish to thank this center for the sincere cooperation. This paper has been derived from student thesis number 925 registered at the faculty of the dental school of Kerman University of Medical Sciences.

\section{References:}

1.Hussain M. Essentials of medical history taking in dental patients. Dent Update 2015; 42(7):687.

2.Katsoulis J, Huber S, Mericske-Stern R. Gerodontology consultation in geriatric facilities: general health status (I). Schweiz Monatsschr Zahnmed 2009;119(1):12-8.

3.Ireland RS, Bowyer V, Ireland A, Sutcliffe P. The medical and dental attendance pattern of patients attending dental practices in Warwickshire and their general health risk assessment. Br Dent J 2012; 20;212(8):E12.

4.Parirokh M, Eghbal MJ, Ghoddusi J, Kakoei S, Haghdoost AA, Kakooei S .The frequency of medically compromised patients in endodontic offices in Iran . Iran Endod J 2013;8(2):48-51.

5.Dhanuthai K, Sappayatosok K, Bijaphala P, Kulvitit S, Sereerat T. Prevalence of medically compromised conditions in dental patients. Med Oral Patol Oral Cir Bucal 2009;14(6):E287-91.

6.Fernández-Feijoo J, Garea-Gorís R, Fernández-Varela M, Tomás-Carmona I, Diniz-Freitas M, LimeresPosse J. Prevalence of systemic diseases among patients requesting dental consultation in the public and private systems. Med Oral Patol Oral Cir Bucal 2012;17(1):e89-93.

7.Brown RS, Farquharson AA, Pallasch TM. Medical consultation for medically complex patients. J Calif Dent Assoc 2007;35(3):343-9.

8.Brasher WJ, Rees TD. The medical consultation: its role in dentistry. J Am Dent Assoc 1977;95(5):961-4.
9.Greenwood M, Meechan JG. General medicine and surgery for dental practitioners: part 3. Management of specific medical emergencies in dental practice. $\mathrm{Br}$ Dent J 2014;217(1):21-6.

10. Skapetis T, Gerzina T, Hu W. Management of dental emergencies by medical practitioners: recommendations for Australian education and training. Emerg Med Australas 2011;23(2):142-52.

11. Greenwood M. Medical emergencies in dental practice. Prim Dent J 2014;3(1):4-5.

12. Anders PL, Comeau RL, Hatton M, Neiders ME. The nature and frequency of medical emergencies among patients in a dental school setting. J Dent Educ 2010; 74(4):392-6.

13. Glick M, Greenberg MS, Jontell M, Ship JA. Introduction to oral medicine: evaluation of the dental patient. In: Glick M, editor. Burket's oral medicine. 12th ed. Shelton, CT: PMPH-USA, LTD; 2015.p.1-16. 14. Laurence B. Dentists consider medical screening important and are willing to incorporate screening procedures into dental practice. J Evid Based Dent Pract 2012; 12(3 Suppl):32-3.

15. Cottrell DA, Reebye UN, Blyer SM, Hunter MJ, Mehta N. Referral patterns of general dental practitioners for oral surgical procedures. J Oral Maxillofac Surg 2007; 65(4):686-90.

16. Jainkittivong A, Yeh CK, Guest GF, Cottone JA. Evaluation of medical consultations in a predoctoral dental clinic. Oral Surg Oral Med Oral Pathol Oral Radiol Endod 1995; 80(4):409-13.

17. Hatipoğlu MG, Hatipoğlu H. Evaluation of Medical Consultations Forms of a Patient Group Admitted to the Dental Clinic of a University Hospital. Ankara Med J 2015;15(1):10-5.

18. Jainkittivong A, Aneksuk V, Langlais RP. Medical health and medication use in elderly dental patients. J Contemp Dent Pract 2004;5(1):31-41.

19.Geist SM, Geist JR. Improvement in medical consultation responses with a structured request form.J Dent Educ 2008; 72(5):553-61.

20. Ahsen NF, Batul SA, Ahmed AN, Imam SZ, Iqbal H, Shamshair K, Ali H. Developing counseling skills through pre-recorded videos and role play: a pre- and post-intervention study in a Pakistani medical school. BMC Med Educ 2010;10(1):7. 